\title{
Theoretical analysis of dynamic force spectroscopy experiments on ligand-receptor complexes
}

\author{
M. Raible ${ }^{a}$, M. Evstigneev ${ }^{a}$, P. Reimann ${ }^{a, *}$, F.W. Bartels ${ }^{b}$, R. Ros ${ }^{b}$ \\ a Theoretische Physik, Universität Bielefeld, Universitätsstrasse 25, 33615 Bielefeld, Germany \\ ${ }^{\mathrm{b}}$ Experimentelle Biophysik, Universität Bielefeld, 33615 Bielefeld, Germany
}

Received 15 December 2003; received in revised form 19 March 2004; accepted 1 April 2004

\begin{abstract}
The forced rupture of single chemical bonds in biomolecular compounds (e.g. ligand-receptor systems) as observed in dynamic force spectroscopy experiments is addressed. Under the assumption that the probability of bond rupture depends only on the instantaneously acting force, a data collapse onto a single master curve is predicted. For rupture data obtained experimentally by dynamic AFM force spectroscopy of a ligand-receptor bond between a DNA and a regulatory protein we do not find such a collapse. We conclude that the above mentioned, generally accepted assumption is not satisfied and we discuss possible explanations.
\end{abstract}

Keywords: Single molecule force spectroscopy; Chemical reaction kinetics

\section{Introduction}

The specific binding of a ligand molecule to a receptor protein is an essential functional principle of molecular recognition processes and many biotechnological applications. The invention (Binnig et al., 1986) and continuous refinement (Meyer and Amer, 1988; Alexander et al., 1989) of atomic force microscopy (AFM) together with other related techniques (see Section 2 in Merkel, 2001) has led to a tremendous progress in our abilities to experimentally observe such single molecule binding and dissociation events (Florin et al., 1994; Dammer et al., 1996; Rief and Grubmüller, 2001). The interpretation of the

\footnotetext{
* Corresponding author. Tel.: +49-521-106-6206; fax: +49-521-106-6455.

E-mail address: reimann@ @physik.uni-bielefeld.de (P. Reimann).
}

experimental data represents a theoretical challenge of foremost importance not only for our understanding of the basic biophysical principles at work but also for the purpose of controlling and manipulating the stability and decay of specific chemical bonds in biotechnological contexts.

The basic principle of dynamic AFM force spectroscopy is schematically sketched in Fig. 1: a single chemical bond of interest, e.g. in a ligand-receptor complex, is connected via two linker molecules with the tip of an AFM cantilever and a piezoelectric element. The latter is employed for "pulling down" the attached linker molecule at some constant velocity which in turn leads to an elastic reaction force of the cantilever, determined from the deflection of a laser beam. The main quantity of interest is the magnitude of the force at the moment when the bond breaks. 


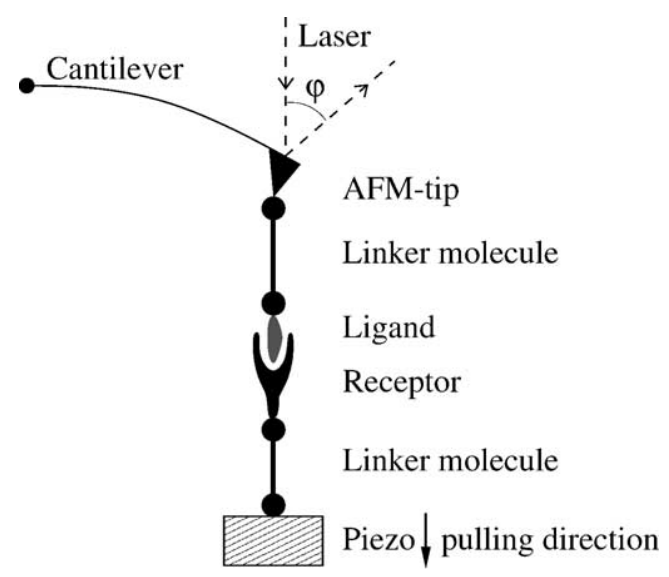

Fig. 1. Schematic illustration of the basic principle of dynamic AFM force spectroscopy.

Important examples of such AFM spectroscopic investigations of molecular compounds with a "small" ligand "docking" into a binding pocket of a "large" protein macromolecule (receptor) include the receptors avidin or streptavidin and the ligands biotin or biotin analoga (Florin et al., 1994; Lee et al., 1994; Moy et al., 1994; Chilcotti et al., 1995; Lo et al., 1995) and antigen-antibody complexes (Dammer et al., 1996; Hinterdorfer et al., 1996; Allen et al., 1997; Schlesinger et al., 2000), to name but a few.

The theoretical interpretation of the observed rupture forces is a non-trivial task: upon repeating the same experiment with the same pulling velocity, the rupture forces are found to be distributed over a wide range. Furthermore, for different pulling velocities different such distributions are obtained. Hence, neither a single rupture event nor the average rupture force at any fixed pulling velocity can serve as a meaningful characteristics quantity of a given chemical bond strength. The main breakthrough in solving the puzzle came with the hallmark 1997 paper by Evans and Ritchie-a precursor being due to Bell 1978-recognizing that a forced bond rupture event is a thermally activated decay of a metastable state that can be described within the general framework of Kramers reaction rate theory (Kramers, 1940; Hänggi et al., 1990; Fleming and Hänggi, 1993; Talkner and Hänggi, 1995).

While Evans and Ritchie's original theoretical approach has been extended and refined in several im- portant directions (Rief et al., 1998; Shillcock and Seifert, 1998; Merkel et al., 1999; Strunz et al., 2000; Heymann and Grubmüller, 2000; Seifert, 2000; Evans, 2001; Nguyen-Duong et al., 2003), the essential physical picture has remained unchanged and has been the basis for evaluating the observed rupture data of all experimental investigations ever since (Merkel, 2001; Evans, 2001). In our present work, we critically reconsider the two most basic assumptions of this standard approach. In Section 2, we carefully discuss the content of these two fundamental assumptions. In Section 3 , we draw our main conclusion, namely a collapse onto a single master curve for a quantity which follows immediately from the experimentally observed rupture data. In Section 4, we discuss as an application of this collapse an optimized method of data analysis and illustrate it for an "idealized" numerically generated set of rupture data in Section 5. In Section 6, we proceed to "real" experimentally obtained rupture data, finding that they are incompatible with one of the two fundamental assumptions from Section 2. In Section 7, possible reasons for this incompatibility are addressed.

\section{Fundamental assumptions}

\subsection{First assumption}

Following Evans and Ritchie (1997), a rupture event is viewed (Merkel, 2001; Evans, 2001) as a thermally activated decay of a metastable state governed by a reaction kinetics of the form

$\frac{\mathrm{d} p(t)}{\mathrm{d} t}=-k(f(t)) p(t)$,

where $p(t)$ is the probability of bond survival up to time $t$ and $k(f)$ the rate of decay in the presence of a pulling force $f$.

Bond re-formation in the presence of the force $f$ is exponentially suppressed and therefore neglected in Eq. (1) (Merkel, 2001; Evans, 2001). The only further assumption implicit in (1) is that the probability of bond rupture depends only on the instantaneously acting force, not on the past history. In particular, an independence on the force protocol $f\left(t^{\prime}\right)$ for times $t^{\prime}<t$ is assumed, including the instantaneous "speed" $\mathrm{d} f(t) / \mathrm{d} t$. The justification is that intramolecular ther- 
mal relaxation processes into the "accompanying" metastable equilibrium are much faster than the time scale on which the applied force $f(t)$ significantly changes (Evans and Ritchie, 1997, 1999; Merkel, 2001; Evans, 2001).

Describing the dissociation of a chemical bond in the absence of an external force $f$ in terms of a reaction rate across some activation barrier is very common and well established. In the same spirit, it is indeed suggestive that also in the presence of an external bias force such a rate description remains valid at least for not too strong and not too quickly varying forces. In particular, such a rate description is by no means restricted to processes that involve only a single relevant chemical reaction coordinate (Hänggi et al., 1990).

\subsection{Second assumption}

The instantaneous force $f(t)$ acting on the chemical bond according to (1) is assumed to depend solely on the instantaneous position $s(t)$ of the piezo's upper surface in Fig. 1, i.e. there exists a function $f_{0}(s)$, called the force-extension characteristics, such that

$f(t)=f_{0}(s(t))$.

Here and in the following, the origin of time and space is tacitly chosen such that at $t=0$ we have $s=0$ and $f=0$. In the most common case, to which we restrict ourselves throughout this paper, the position $s$ of the piezo moves "downward" with some constant velocity $v$, i.e.

$s=v t$.

In this case, the basic assumption in (2) is tantamount to the independence of the force-extension characteristics $f_{0}(s)$ on the pulling velocity $v$.

This assumption is by no means obviously satisfied in a real experimental system. First of all, it may be difficult to carry out the required large number of rupture measurements with always the same linker molecules and the same cantilever. Therefore it is not obvious that the elastic properties of cantilever and linkers in Fig. 1 are (practically) identical even for a subset of rupture measurements obtained at the same pulling velocity $v$. Second, when proceeding to increasingly larger pulling velocities, there is no a priori reason why the linker molecules should not behave, for instance, in an increasingly "stiffer" way.

On the other hand, the assumption can be easily checked by verifying that the deflection of the laser beam in Fig. 1 solely depends on the instantaneous position $s(t)$ of the piezo, i.e. does not change upon repeating the same experiment nor upon changing the pulling velocity $v$. In doing so, one exploits that the force acting on the chemical bond is identical to the elastic force of the cantilever due to Newtons law "actio=reactio". Indeed, the applied force cannot get "lost" or "internally dissipated" since friction caused by the environmental fluid and inertia effects of all the moving pieces in Fig. 1 can safely be neglected under realistic experimental conditions (Evans, 2001).

Note that in general the force $f_{0}(s)$ will be a nonlinear function of the elongation $s$ due to a non-linear force-extension characteristics (Evans and Ritchie, 1999) of one or several of the elastic elements (cantilever, linkers, ligand, receptor) acting in series in Fig. 1.

We finally remark that in order to extract interesting information out of experimentally measured rupture data, one usually needs to make further assumptions on the functional form of the rate $k(f)$ in (1) (Merkel, 2001; Evans and Ritchie, 1997; Evans, 2001). However, for the main part of our present paper, no such additional assumptions are required.

\section{Main conclusion}

Due to the postulated independence of $f_{0}(s)$ on how $s$ changes in time, we may temporally consider $s$ as a time-independent parameter to conclude that the force $f_{0}(s)$ is a strictly monotonic function of the displacement $s$. Hence the inverse function $f_{0}^{-1}(f)$ is well defined for all forces $f$ that may play a role in a real experiment. Consequently, by introducing (2) and (3) into (1) and then going over from time $t$ to force $f$ as independent variable, one can infer that

$\frac{\mathrm{d} p_{v}(f)}{\mathrm{d} f}=-\frac{1}{v} \frac{k(f)}{f_{0}^{\prime}\left(f_{0}^{-1}(f)\right)} p_{v}(f)$,

where $p_{v}(f(t)):=p(t)$ is the probability of bond survival up to a pulling force $f$ at an arbitrary but fixed pulling velocity $v$. With the initial condition $p(t=$ 
$0)=p_{v}(f=0)=1$, the differential equation (4) is readily integrated, yielding

$p_{v}(f)=\left\{\exp -\frac{g(f)}{v}\right\}$

where we have introduced

$g(f):=\int_{0}^{f} \mathrm{~d} f^{\prime} \frac{k\left(f^{\prime}\right)}{f_{0}^{\prime}\left(f_{0}^{-1}\left(f^{\prime}\right)\right)}$.

Eqs. (5) and (6) immediately lead us to the main conclusion of our present paper: under the assumptions expressed by Eqs. (1)-(3) the quantity $-v \ln p_{v}(f)=$ $g(f)$ is independent of the pulling velocity $v$. Since the probability $p_{v}(f)$ of bond survival up to a pulling force $f$ readily follows from the experimentally observed rupture events (see also Eq. (7) below), the same applies to the quantity $-v \ln p_{v}(f)=g(f)$. In other words, when plotting the functions $-v \ln p_{v}(f)$ for several different pulling velocities $v$, a data collapse onto a single master curve - up to experimental and statistical inaccuracies-is predicted.

Once this master curve $g(f)$ is (approximately) determined, the decay rate $k(f)$ as a function of the acting force $f$ immediately follows by differentiating Eq. (6), provided the force-extension characteristics $f_{0}(s)$ has been experimentally determined and is indeed $v$-independent, cf. Section 2. On the other hand, if the observed data do not collapse onto a single master curve then it follows that one of the two fundamental assumptions from Section 2 is violated.

\section{Optimized data analysis}

Given a set of $N_{v}$ rupture forces $f_{n}, n=1, \ldots, N_{v}$, at a fixed pulling velocity $v$, the best estimate for $p_{v}(f)$ that can be inferred from these data is

$\tilde{p}_{v}(f)=\frac{1}{N_{v}} \sum_{n=1}^{N_{v}} \Theta\left(f_{n}-f\right)$,

where $\Theta(x)$ is the Heaviside step function. Here and in the following, a tilde indicates an estimate for the corresponding "true" quantity without tilde, towards which it converges for $N_{v} \rightarrow \infty$. For instance, from (7) one can go on to determine the associated estimate

$\tilde{g}_{v}(f):=-v \ln \tilde{p}_{v}(f)$ for the "true" function $g(f)$ in (5), (6). Let us now consider an arbitrary but fixed $f>0$. Then, for each pulling velocity $v$ an estimate $\tilde{g}_{v}(f)$ for the "true" $g(f)$ follows from Eqs. (7) and (8). Its reliability is quantified by some variance $\sigma_{\tilde{g}_{v}(f)}^{2}$. With this amount of information at our disposition, according to the so called method of weighted averages (Taylor, 1982), the best guess for the "true" $g(f)$ is represented by that argument $x$ which minimizes the weighted sum of square deviations $\sum_{v}\left[x-\tilde{g}_{v}(f)\right]^{2} / \tilde{\sigma}_{\tilde{g}_{v}(f)}^{2}$, where $\sum_{v}$ indicates a summation over all pulling velocities $v$. Referring to Evstigneev and Reimann (2003) for the calculational details, one can show that this best guess for $g(f)$ is given by the weighted average

$\tilde{g}(f)=\sum_{v} c_{v}(f) \tilde{g}_{v}(f)$,

$c_{v}(f):=\frac{N_{v} \tilde{p}_{v}^{2}(f) \mathrm{e}^{\tilde{g}(f) / v}}{v^{2}\left[1-\mathrm{e}^{-\tilde{g}(f) / v}\right]} \sigma_{\tilde{g}(f)}^{2}$

$\sigma_{\tilde{g}(f)}^{2}:=\left(\sum_{v} \frac{N_{v} \tilde{p}_{v}^{2}(f) \mathrm{e}^{\tilde{g}(f) / v}}{v^{2}\left[1-\mathrm{e}^{-\tilde{g}(f) / v}\right]}\right)^{-1}$.

Note that (9) together with (10), (11) represents a transcendental equation for $\tilde{g}(f)$, which needs to be numerically solved. The result is an estimate $\tilde{g}(f)$ for the "true" function $g(f)$ in (6) together with its statistical uncertainty (11).

\section{Illustration for numerically generated data}

In order to illustrate the general concepts from Sections 3 and 4, we generated rupture data by means of a numerical computer simulation of an actual experiment. To this end, we have assumed for the rate $k(f)$ in (1) the following standard functional form (Bell, 1978; Evans and Ritchie, 1997)

$k(f)=k_{0} \exp \{\alpha f\}$

with the typical values of the rupture parameters (Merkel, 2001)

$k_{0}=1 \mathrm{~s}^{-1}, \quad \alpha=0.1 \mathrm{pN}^{-1}$.

Regarding the force-extension characteristics $f_{0}(s)$ in (2), we focussed on the simplest case of a linear relation

$f_{0}(s)=\kappa s$ 


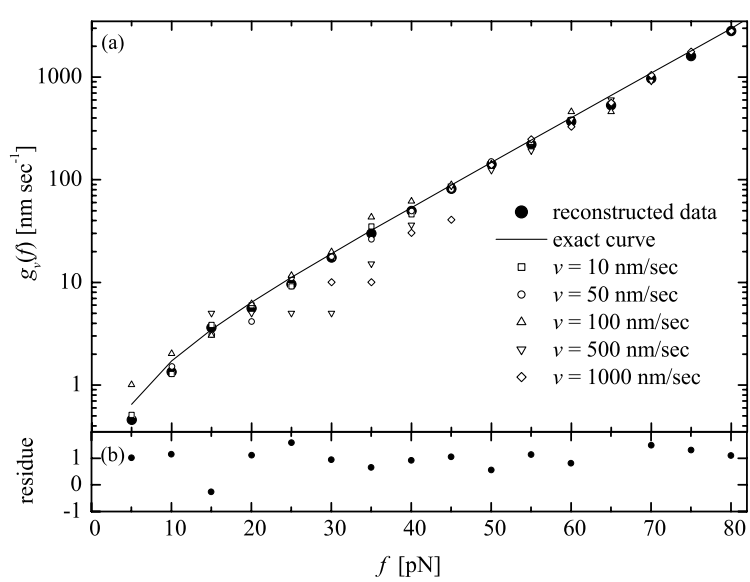

Fig. 2. (a) The function $\tilde{g}_{v}(f)$ vs. force $f$ for five different pulling velocities $v$, obtained according to (7), (8) with numerically generated rupture data as detailed in the main text of Section 5. For the sake of clarity, only a small set of discrete $f$-values are plotted with different symbols for the different pulling velocities $v$. The resulting estimate for $\tilde{g}(f)$ according to (9) is represented as filled circles. The "true" function $g(f)$ from (6) is indicated as solid line. (b) Normalized residues $[\tilde{g}(f)-g(f)] / \sigma_{\tilde{g}(f)}$ vs. force $f$ according to (11).

with a representative elasticity coefficient

$\kappa=10 \mathrm{pN} \mathrm{nm}^{-1}$.

On the basis of (1-3) with (12-15) we have numerically sampled rupture forces $f_{n}, n=1, \ldots, N_{v}$, for different pulling velocities $v$ on the computer. As compared to real experimentally measured rupture forces, these numerically generated data have the advantage (Friedsam et al., 2003; Evstigneev and Reimann, 2003) of being free from other uncertainties inevitably present in a real experiment, and the underlying statistical properties, e.g. the "true" function $g(f)$ in (6), are exactly known. Moreover, the two assumptions from Section 2 are automatically fulfilled.

Along these lines, we have simulated $N_{v}=100$ rupture events for each of five pulling velocities $v=$ $10,50,100,500$, and $1000 \mathrm{~nm} \mathrm{~s}^{-1}$, representing typical values in real experimental investigations (Evans and Ritchie, 1999; Merkel, 2001), and evaluated them according to (7)-(11). The obtained results are depicted in Fig. 2. The functions $\tilde{g}_{v}(f)$ for different pulling velocities $v$ indeed collapse within their statistical uncertainties onto a single master curve.
Also the estimate $\tilde{g}(f)$ for the "true" function $g(f)$ in (6) is rather good over a wide range of pulling forces $f$.

\section{Application to experimental data}

Next we turn to rupture data of the specific ligandreceptor bond between DNA and a regulatory protein observed experimentally by dynamic AFM force spectroscopy. For a more detailed discussion of the experiment itself we refer to the original publication by Bartels et al. (2003), see also p. ... of the present special issue.

In Fig. 3, we show the result of evaluating these experimentally obtained rupture data in exactly the same manner as the numerically generated data in Section 5 . The main conclusion is that the functions $\tilde{g}_{v}(f)$ for different pulling velocities $v$ do not collapse within their statistical and experimental uncertainties onto a single master curve: we are not dealing with some minute divergences but with a disagreement by two orders of magnitude upon variation of the pulling velocity over a similar range. We also evaluated along the same lines the rupture force data on protein A-immunoglobulin G complexes (Nguyen-Duong et al., 2003; Merkel, 2003) with a similar result.

An obvious first guess of why no data collapse to a single master curve is observed is that the second assumption from Section 2, encapsulated by Eq. (2), may be violated. Accordingly, in Fig. 4, we have plotted the experimentally measured force-extension curves for two rather different pulling velocities $v$. As anticipated in Section 2, these force-extension curves are not strictly linear, though the actual non-linearities are rather tame. More importantly, from these curves we conclude that $f_{0}(s)$ is in fact practically identical for different pulling experiments at one and the same velocity $v$. Comparing the curves for different velocities $v$, there are notable but still rather small differences. In particular, the concomitant $v$-dependence of $g(f)$ in (6) could by far not explain the findings in Fig. 3. Therefore, we are bound to conclude that the experimentally observed data in Fig. 3 are incompatible with the assumption expressed by Eq. (1).

We note that an evaluation of the same experimental data along the standard method in this context has been published by Bartels et al. (2003) (see Fig. 4 therein) 


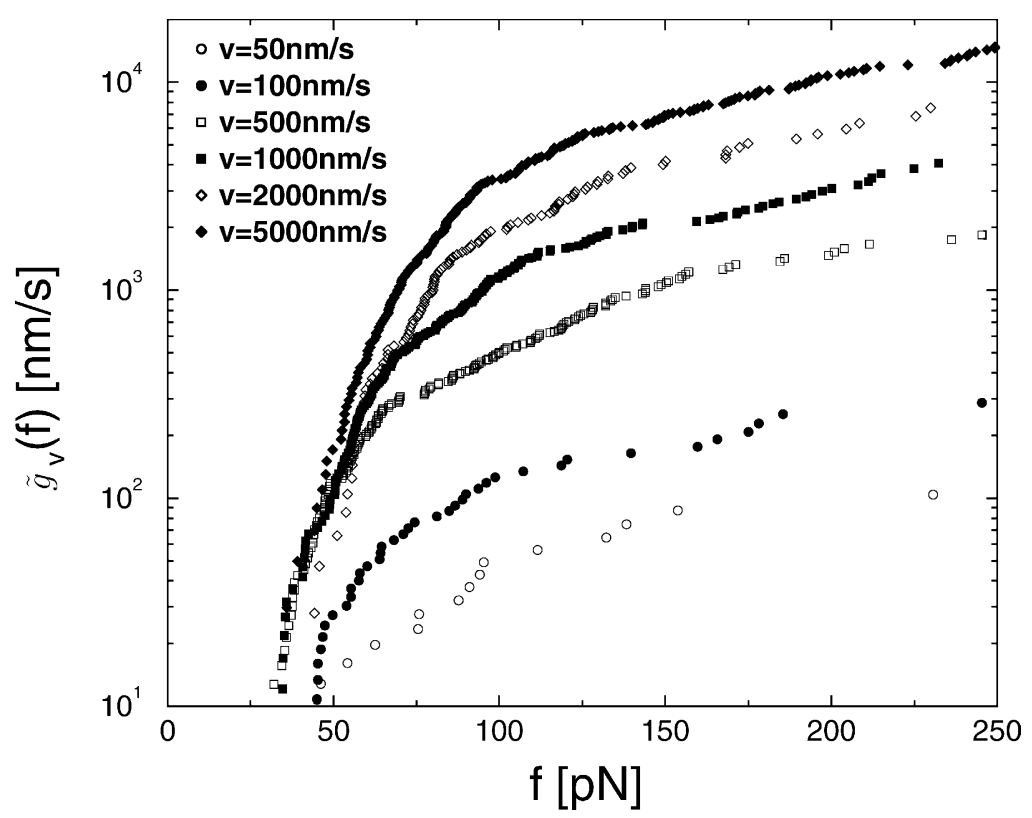

Fig. 3. The functions $\tilde{g}_{v}(f)$ vs. force $f$ for six different pulling velocities $v$. The rupture forces for the DNA fragment expE1/E5 and the regulatory protein ExpG as obtained via dynamic AFM force spectroscopy by Bartels et al. (2003) have been evaluated according to (7), (8). Each depicted point corresponds to one rupture event at $f=f_{n}$ in (7). Accordingly, in between the depicted points, the functions $\tilde{g}_{v}(f)$ in (8) exhibit a trivial, piecewise constant behavior (not shown).

and that in this way the above incompatibility remains undetectable.

\section{Discussion}

To our knowledge, all dynamic force spectroscopy experiments investigating single chemical bond strengths so far rely in their quantitative interpretation of the observed data on the first assumption from Section 2, encapsulated by Eq. (1). Therefore, the resolution of the above reported incompatibility with this assumption is a challenge of foremost importance both theoretically and experimentally. In particular, one has to convincingly explain the following two main features of Fig. 3: (i) For any fixed pulling velocity $v$, the function $\tilde{g}_{v}(f)$ still shows the same typical qualitative features as for the "idealized" numerically generated data in Fig. 2. (ii) With increasing pulling velocity $v$, the curves $\tilde{g}_{v}(f)$ "move upwards" in Fig. 3 . Since the rate law (1) is certainly correct for asymptotically slowly varying forces $f(t)$ (cf. Section 2), this feature translates via (6) into the conclusions that with increasing pulling velocity $v$, bond ruptures become increasingly more likely than predicted by the rate law (1). Presently, we are not yet in the position to propose a convincing resolution of this puzzle. We can only offer a discussion of our so far attempts.

\subsection{Small rupture forces}

Rupture events occurring at very small rupture forces $f$ cannot be distinguished from thermal fluctuations and other artifacts (see Fig. 4) and are therefore missing in the experimental data set. This problem can be circumvented by introducing a threshold $f_{\min }$ and a priori restricting oneself to a statistical ensemble of rupture events that occur at rupture forces larger than $f_{\min }$. The associated modified probability of bond survival up to a pulling force $f$ then satisfies by construction $p_{v}\left(f_{\min }\right)=1$. As a consequence, Eqs. (4) and (5) remain valid for all $f \geq f_{\min }$, while in (6) the lower integration limit has to be replaced by $f_{\min }$. For any preset value of $f_{\min }$ it then still follows that under the assumptions expressed by Eqs. (1)-(3), the quantity $-v \ln p_{v}(f)$ will be independent of the pulling 

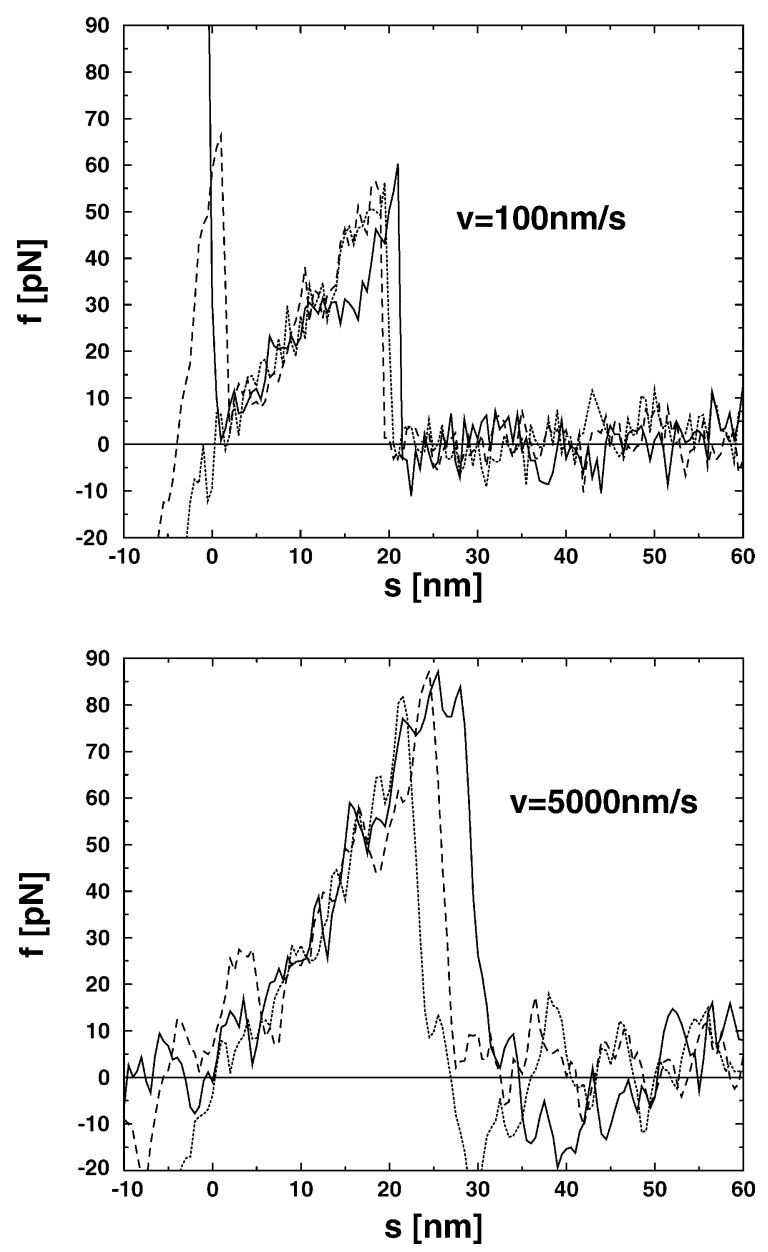

Fig. 4. Parametric plot (with time $t$ as parameter) of the force $f=f(t)$, following from the experimentally observed laser deflection in Fig. 1 and the known elasticity of the cantilever (see also Section 2), vs. the position $s=s(t)$ of the piezo's upper surface for two different pulling velocities $v$ in (3). In each plot, the force-extension curves for three different representative pulling experiments are shown. The erratic fine structures of the observed curves are due to thermal noise and limited experimental accuracy. Bond rupture events are signaled by a sudden force drop to $f=0$. As explained in Section 1, even for experiments conducted at the same pulling velocity, the observed rupture forces may notably differ. Of main interest for us are the increasing parts of the curves prior to bond rupture, associated with the theoretical force-extension characteristics $f_{0}(s)$ from Section 2. To the extent that these parts fall on top of each other, $f_{0}(s)$ can be considered as identical for different pulling experiments, both at the same and at different pulling velocities $v$. velocity $v$ for all $f \geq f_{\min }$. In fact, Fig. 3 has been obtained in this way with $f_{\min }=24 \mathrm{pN}$. By chosing even higher values of $f_{\min }$, such as $f_{\min }=50 \mathrm{pN}$ or $f_{\min }=$ $100 \mathrm{pN}$, we did not observe any clear tendency towards a better data collapse onto a single master curve.

\subsection{Intermediate energy barriers}

In the simplest case, the chemical reaction path underlying the rate law in (1) proceeds from a bound, metastable state across an energy barrier (activated state) towards a dissociated product state. In more general cases, which we cannot exclude a priori, there may exist additional intermediate metastable states separated by additional intermediate energy barriers (Evans and Ritchie, 1997; Merkel et al., 1999; Strunz et al., 2000). It is not immediately clear whether such additional local minima and maxima along the reaction path might lead under experimentally realistic conditions to a breakdown of the assumption expressed by (1) or not. However, if such a breakdown should occur, then this would only be possible in a way that with increasing pulling velocity, bond ruptures become increasingly less probable than predicted by the rate law (1), in contrast to the above mentioned experimentally observed feature (ii). The reason is a follows: even in the presence of intermediate energy barriers, for every fixed value of the force $f$ which possibly may play a role in the experiment, a statistical ensemble of decay processes will approach a quasi-steady probability distribution within that part of the state space (reaction path) which describes the not yet dissociated chemical bond. Its integral is identical to the probability of bond survival up to time $t$ and satisfies a rate law of the form (1) but with a fixed value of the force $f$. Furthermore, the associated parametrically $f$-dependent decay rate $k(f)$ will of course be an increasing function of the force $f$ that is applied to the chemical bond. Turning to the case that the force $f(t)$ increases with time, the only way that the validity of the accompanying instantaneous rate law (1) can be violated is that the probability distribution of not yet dissociated bonds does not have enough time to settle down to the accompanying quasi-steady distribution. Rather, it will "lag behind" that instantaneous quasi-steady distribution and hence the instantaneous decay probability will be smaller than predicted by (1). 


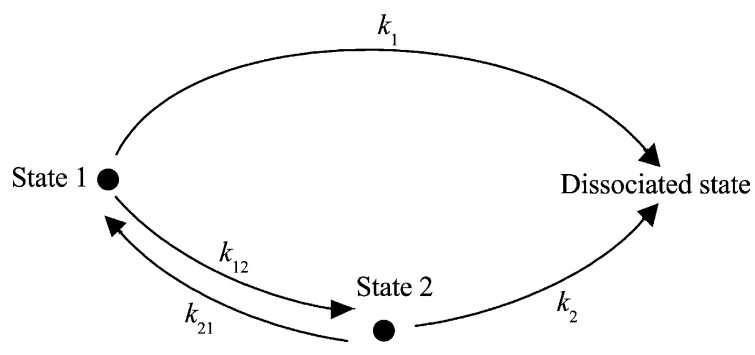

Fig. 5. Schematic representation of a chemical decay that may proceed along two different reaction pathways-one with an intermediate metastable state-into the dissociated state.

\subsection{Several reaction pathways}

A natural extension of the viewpoint put forward in the preceding Section 7.2 is to assume that the decay process of the chemical bond may not always proceed along the same reaction path (Balsera et al., 1997; Paci et al., 2001). Restricting ourselves to the simplest case (Bartolo et al., 2002), we assume that there are just two different reaction pathways of relevance. Furthermore, along each of them there may exist additional intermediate metastable states. A specific example is depicted in Fig. 5. Each metastable state is labeled by an index $i$ and is characterized by its instantaneous population $p_{i}(t)$. Transitions from state $i$ to state $j$ are described by a rate $k_{i j}(f)$ which in general depends on the force $f$. Similarly, transitions from a metastable state $i$ into the dissociated state (bond rupture) are described by a rate $k_{i}(f)$, while recombination reactions from the dissociated state back to a metastable bound state are neglected (Evans, 2001). All together, the decay of any state $i$ is then governed by a reaction kinetics of the form

$$
\begin{aligned}
\frac{\mathrm{d} p_{i}(t)}{\mathrm{d} t}= & -\left[k_{i}(f(t))+\sum_{j \neq i} k_{i j}(f(t))\right] p_{i}(t) \\
& +\sum_{j \neq i} k_{j i}(f(t)) p_{j}(t) .
\end{aligned}
$$

Our first remark is that within such a description we implicitly extend the first assumption from Section 2 in so far as now each rate appearing in (16) is assumed to depend only on the instantaneously acting force $f(t)$. The justification is (Evans and Ritchie, 1997; Merkel, 2001; Evans, 2001) that intramolecular thermal relax- ation processes into the "accompanying" quasi-steady distribution within each separate metastable state are much faster than the time scale on which the applied force $f(t)$ significantly changes (Evans and Ritchie, 1999). The salient difference with (1) and its justification is that now we have already separated out all possibly "dangerous" slow processes-namely transitions between metastable states-from the truly very fast relaxation processes within each metastable state. Therefore, the present assumption is much weaker and can safely be taken for granted for all experimentally realistic forces $f(t)$. In fact, the only case known to us in which this assumption becomes problematic are heme proteins at low temperatures, where they exhibit "glass-like" properties (Frauenfelder and Wolynes, 1985; Frauenfelder et al., 1991).

For any fixed value of the force $f$, the full kinetics (16) can be reduced (Strunz et al., 2000), after initial transients have died out, to an effective single-rate law of the form (1) with

$p(t)=\sum_{i} p_{i}(t)$

However, for sufficiently quickly varying forces $f(t)$ such a reduction is no longer possible since the transitions between different states are then too slow to let the distribution of probabilities among these states settle down to their accompanying quasi-steady state values. Put differently, the above mentioned transients have no time to die out.

In short, an extended reaction kinetics (16) allows us to quantitatively explore the range of validity of the assumption expressed by (1) and, in case it breaks down, to go in a physically meaningful way beyond it. We also note that the situation discussed in the preceding Section 7.2 is recovered as a special case, namely several metastable states arranged along a single reaction path.

Concerning initial conditions, it is natural to assume thermal equilibrium among the metastable states and to assign probability zero to the dissociated state. The former condition is tantamount to detailed balance symmetry (Hänggi et al., 1990), i.e.

$p_{i}(0) k_{i j}(0)=p_{j}(0) k_{j i}(0)$ 
for all $i, j$, and the latter to the normalization

$\sum_{i} p_{i}(0)=1$.

Regarding the force dependence of the rates $k_{i j}(f)$ and $k_{i}(f)$, we focus on the same standard functional form (Bell, 1978; Evans and Ritchie, 1997) as in (12), i.e.

$k_{i j}(f)=k_{i j}(0) \exp \left\{\alpha_{i j} f\right\}, \quad k_{i}(f)=k_{i}(0) \exp \left\{\alpha_{i} f\right\}$.

As can be seen from Fig. 4, the force-extension characteristics $f_{0}(s)$ is not perfectly linear, but still very well approximated by a linear behavior of the form (cf. (14))

$f_{0}(s)=\kappa s, \quad \kappa=3 \mathrm{pN} \mathrm{nm}^{-1}$.

Focusing on the specific example from Fig. 5, we have numerically determined bond survival probabilities $p_{v}(f(t))=p(t)$ up to a pulling force $f=f(t)$ according to (16)-(21) for various realistic sets of parameters $k(0)$ and $\alpha$ in (20). The main qualitative features of the resulting functions $g_{v}(f):=-v \ln p_{v}(f)$ were always the same. Hence, we restrict ourselves to the parameter set that came closest to the experimentally observed results in Fig. 3, namely

$k_{12}(0)=k_{21}(0)=k_{1}(0)=k_{2}(0)=0.3 \mathrm{~s}^{-1}$

$\alpha_{12}=\alpha_{21}=0 \mathrm{pN}^{-1}, \quad \alpha_{1}=0.1 \mathrm{pN}^{-1}$,

$\alpha_{2}=0.02 \mathrm{pN}^{-1}$,

i.e. without external force the ligand-receptor complex has two equivalent "internal states", while under force their "internal" transition rates remain unaffected but their individual dissociation rates become different. The resulting functions $g_{v}(f)$ for the same pulling velocities as in Fig. 3 are depicted in Fig. 6. While there are many similarities between the two figures, there remain significant discrepancies. As already mentioned, these discrepancies can not be further diminished by choosing other parameters than in (22) and (23).

In conclusion, taking into account several reaction pathways does indeed lead to a violation of the assumption expressed by (1) and reproduces nicely several of the experimentally observed main features, but still does not satisfactorily explain all of them. In par-

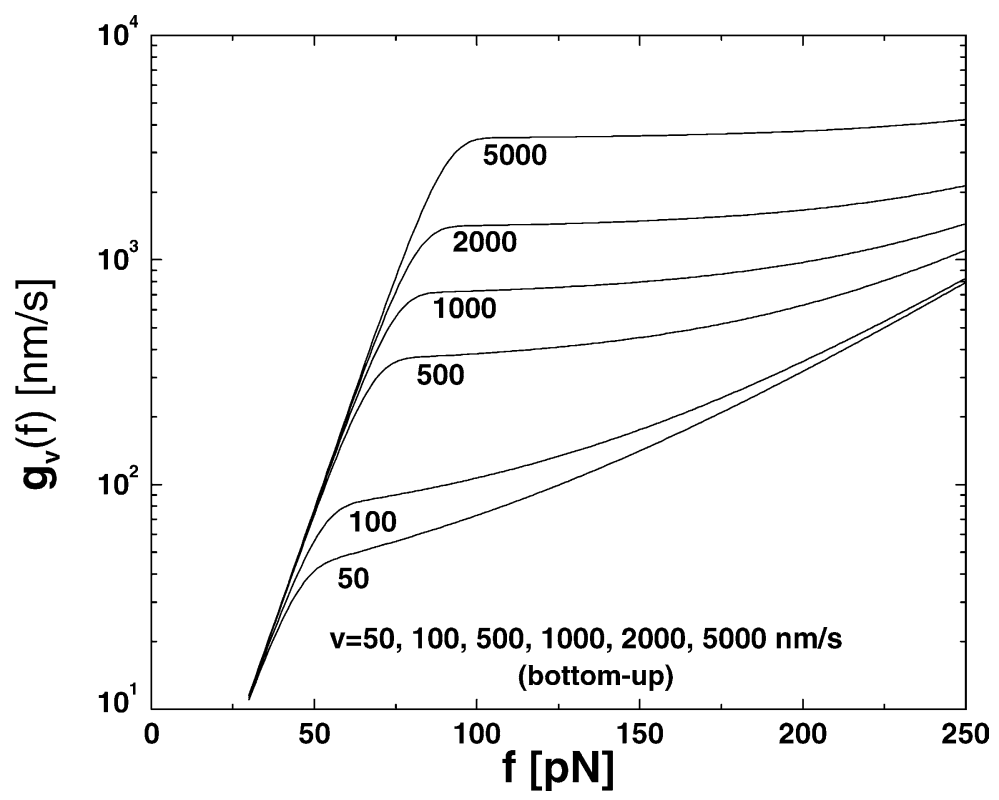

Fig. 6. The functions $g_{v}(f):=-v \ln p_{v}(f)$ vs. force $f$ for the same pulling velocities $v$ as in Fig. 3. Shown are results for the kinetic reaction scheme from Fig. 5 as detailed in the main text of Section 7.3. 
ticular, the convergence of the curves at large forces in Fig. 6 is absent in Fig. 3.

\section{Acknowledgements}

Helpful discussions with R. Eckel, D. Anselmetti, R. Eichhorn, and especially R. Merkel are gratefully acknowledged. This work was supported by Deutsche Forschungsgemeinschaft under SFB 613 (Teilprojekt K7), the Alexander von Humboldt-Stiftung, and the ESF-program STOCHDYN.

\section{References}

Alexander, S., Hellemans, L., Marti, O., Schneir, J., Elings, V., Hansma, P.K., Longmire, M., Gurley, J., 1989. An atomic-resolution atomic-force microscope implemented using an optical lever. J. Appl. Phys. 65, 164-167.

Allen, S., Chen, X., Davies, J., Davies, M.C., Dawkes, A.C., Edwards, J.C., Roberts, C.J., Sefton, J., Tendler, S.J.B., Williams, P.M., 1997. Detection of antibody-antigen binding events with atomic force microscope. Biochemistry 36, 7457 7463.

Balsera, M., Stepaniants, S., Izrailev, S., Oono, Y., Schulten, K., 1997. Reconstructing potential energy functions from simulated force-induced unbinding processes. Biophys. J. 73, 12811287.

Bartels, F.W., Baumgarth, B., Anselmetti, D., Ros, R., Becker, A., 2003. Specific binding of the regulatory protein ExpG to promotor regions of the galactoglucan biosynthesis gene cluster of Sinorhizobium meliloti-a combined molecular biology and force spectroscopy investigation. J. Struct. Biol. 143, 145.

Bartolo, B., Derényi, I., Ajdari, A., 2002. Dynamic response of adhesion complexes: beyond the single-path picture. Phys. Rev. E 65, 051910 .

Bell, G.I., 1978. Models for the specific adhesion of cells to cells. Science 200, 618-627.

Binnig, G., Quate, C.F., Gerber, C., 1986. Atomic force microscope. Phys. Rev. Lett. 56, 930-933.

Chilcotti, A., Boland, T., Ratner, B.D., Stayton, P.S., 1995. The relationship between ligand-binding thermodynamics and protein-ligand interaction forces measured by atomic force microscopy. Biophys. J. 69, 2125-2130.

Dammer, U., Hegner, M., Anselmetti, D., Wagner, P., Dreier, M., Huber, W., Güntherodt, H.-J., 1996. Specific antigen/antibody interaction measured by force microscopy. Biophys. J. 70, 2437-2441.

Evans, E., Ritchie, K., 1997. Dynamics strength of molecular adhesion bonds. Biophys. J. 72, 1541-1555.

Evans, E., Ritchie, K., 1999. Strength of a weak bond connecting flexible polymer chains. Biophys. J. 76, 2439-2447.
Evans, E., 2001. Probing the relation between force, lifetime, and chemistry in single molecular bonds. Annu. Rev. Biomol. Struct. 30, 105-128.

Evstigneev, M., Reimann, P., 2003. Dynamic force spectroscopy: optimized data analysis. Phys. Rev. E 68, 045103(R).

Fleming, G.R., Hänggi, P. (Ed.), 1993. Activated Barrier Crossing. World Scientific, Singapore.

Florin, E.-L., Moy, V.T., Gaub, H.E., 1994. Adhesion forces between individual ligand-receptor pairs. Science 264, 415417.

Frauenfelder, H., Wolynes, P.G., 1985. Rate theories and puzzles of hemeprotein kinetics. Science 229, 337.

Frauenfelder, H., Sligar, S.G., Wolynes, P.G., 1991. The energy landscapes and motions of proteins. Science 254, 1598.

Friedsam, C., Wehle, A.K., Kühner, F., Gaub, H.E., 2003. Dynamic single-molecule force spectroscopy: bond rupture analysis with variable spacer length. J. Phys.: Condens. Matter 15, S1709S1723.

Hänggi, P., Talkner, P., Borkovec, M., 1990. Reaction-rate theory: fifty years after Kramers. Rev. Mod. Phys. 62, 251-342.

Heymann, B., Grubmüller, H., 2000. Dynamic force spectroscopy of molecular adhesion bonds. Phys. Rev. Lett. 84, 6126-6129.

Hinterdorfer, P., Baumgartner, W., Gruber, H.J., Schilcher, K., Schindler, H., 1996. Detection and localization of individual antibody-antigen recognition events by atomic force microscopy. Proc. Natl. Acad. Sci. U.S.A. 93, 3477-3481.

Kramers, H.A., 1940. Brownian motion in a field of force and the diffusion model of chemical reactions. Physica (Utrecht) 7, 284-304.

Lee, G.U., Kidwell, A.D., Colton, R.J., 1994. Sensing discrete streptavidin-biotin interactions with atomic force microscopy. Langmuir 94, 354-357.

Lo, Y.-S., Zhu, Y.-J., Beebe Jr., T.B., 1995. Loading-rate dependence of individual ligand-receptor bond-rupture studied by atomic force microscopy. Langmuir 17, 3741-3748.

Merkel, R., Nassoy, P., Leung, A., Ritchie, K., Evans, E., 1999. Energy landscapes of receptor-ligands bonds explored with dynamic force spectroscopy. Nature 397, 50-53.

Merkel, R., 2001. Force spectroscopy on single passive biomolecules and single biomolecular bonds. Phys. Rep. 346, 343385.

Merkel, R., 2003, private communication.

Meyer, G., Amer, N.M., 1988. Novel approach to atomic force microscopy. Appl. Phys. Lett. 53, 1045-1047.

Moy, V.T., Florin, E.-L., Gaub, H.E., 1994. Intramolecular forces and energies between ligands and receptors. Science 266, 257259.

Nguyen-Duong, N., Koch, K.W., Merkel, R., 2003. Surface anchoring reduces the lifetime of single specific bonds. Europhys. Lett. 61, 845.

Paci, E., Caflisch, A., Plückthun, A., Karplus, M., 2001. Forces and energetics of hapten-antibody dissociation: a biased molecular dynamics simulation study. J. Mol. Biol. 314, 589-605.

Rief, M., Fernandez, J.M., Gaub, H.E., 1998. Elastically coupled two-level systems as a model for biopolymer extensibility. Phys. Rev. Lett. 81, 4764-4767. 
Rief, M., Grubmüller, H., 2001. Kraftspektroskopie von einzelnen Biomolekülen. Phys. B1. 57 (2), 55.

Schwesinger, F., Ros, R., Strunz, T., Anselmetti, D., Güntherodt, H.-J., Honegger, A., Jermutus, L., Tiefenauer, L., Plückthun, A., 2000. Unbinding forces of single antibody-antigen complexes correlated with their thermal dissociation rates. Proc. Natl. Acad. Sci. U.S.A. 97, 9972-9977.

Seifert, U., 2000. Rupture of multiple molecular bonds under dynamic loading. Phys. Rev. Lett. 84, 2750-2753.
Shillcock, J., Seifert, U., 1998. Escape from a metastable well under a time-ramped force. Phys. Rev. E 57, 7301-7304.

Strunz, T., Oroszlan, K., Schumakovitch, I., Güntherodt, H.-J., Hegner, M., 2000. Model energy landscapes and the force-induced dissociation of ligand-receptor bonds. Biophys. J. 79, 1206-1212.

Talkner, P., Hänggi, P. (Eds.), 1995. New Trends in Kramers Reaction Rate Theory. Kluwer, Dordrecht.

Taylor, J.R., 1982. An Introduction to Error Analysis. Mill Valley. 